\title{
Polyvinyl Chloride Insoluble Gels: Their Structure and Effect on Properties of Cast Films
}

\author{
F. E. FILISKO \\ Department of Materials and Metallurgy \\ The University of Michigan \\ Ann Arbor, Michigan
}

The effect of insoluble gel material, crystalline aggregates, and suspension particle membranes on the physical and mechanical properties of solution cast films of suspension PVC has been studied. The chemical structure and morphology of the insoluble gel material was also studied. The suspension particle membranes are found to contain significant amounts of hydroxyl and carbonyl groups but are still predominately PVC. These particles affect the transparency of cast films as well as the surface topography by forming discrete clusters upon drying which are uniformly distributed throughout the films. Finally, insoluble gel material is found to have no significant effect on the low and high speed mechanical properties of the films.

\section{INTRODUCTION}

$\mathbf{T}$ he existence of insoluble material or gels in solutions of PVC is well recognized (1-3). In suspension polymerized PVC, however, there are two distinct types of insoluble gel material that appear when the polymer is dissolved. The first type is the so called "crystalline aggregates" which are presumably clusters of PVC molecules bound together by crystalline intermolecular crosslinks. The second type, which will be referred to as "suspension particle membranes" (SPM), are seemingly a consequence of the method of polymerization and the suspension medium. They develop as thin membranes surrounding the individual particles during suspension polymerization (4). These membranes are insoluble in any known PVC solvent and remain insoluble even at the boiling temperature of cyclohexanone.

For viscosity and osmotic pressure measurements (5-7), SPM gels can be removed by filtering and ultracentrifugation. To what extent the crystalline aggregates are removed by these procedures depends on filter pore size or centrifugation speed. The total amount of insoluble material removed is usually of the order of 0.01-0.1 percent by weight. In many of the measurements on bulk materials, such as mechanical measurements, thermal spectroscopy, infrared, melt viscosity, etc., the presence of the SPM gels has been considered to have no effect on the resultant measurements, the reason being that there is no way to remove this SPM material without dissolving the polymer and no way to determine the amount of gel material in the bulk samples, although a possible method related to the presence of infrared absorption bands due to the SPM particles is presented here.

In this study the effects of the presence of the SPM particles are determined on the physical and mechanical properties of solution-cast films of suspension PVC. Likewise, the chemical structure and morphology of the SPM particles is investigated. A wide variation in mechanical properties is known to exist between solution-cast samples, extruded samples and compression-molded samples as studied by Berens and Folt $(8,9)$. The effect of molecular weight and its distribution, as well as syndiotacticity, on mechanical properties is also significant. Therefore it should be emphasized that we are specifically looking at the effects of SPM particles on physical and mechanical properties of solution-cast films. It is necessary to use solution-cast films in order to vary the amounts of SPM while keeping all other variables such as molecular weight, molecular weight distribution, heat history, syndiotacticity, and method of sample preparation constant.

\section{EXPERIMENTAL}

Three unstabilized resins were used in the study; a B.F. Goodrich resin, a General resin, and a Diamond resin. All were suspension polymerized with polyvinyl alcohol as the suspension agent.

Separation of gels was accomplished by dissolving the resins in tetrahydrofuran at concentrations of less than 2 percent by weight. The solutions were then heated to $60^{\circ} \mathrm{C}$ for about 1 hour and cooled. The gels were separated in one of two ways. One method was by putting the solutions in tall graduated cylinders and allowing them to remain undisturbed for a period of one week to a few months. The gels would settle to the bottom of the containers. Most of the aggregates would settle out in one week while others would remain suspended indefinitely. The second method was by centrifuging the 2 percent solutions at speeds of around $8000 \mathrm{rpm}$. The insoluble material would then form a gelatinous 
precipitate on the bottom of the centrifuge tubes that could easily be redispersed. The gels were concentrated by a series of separations and redispersions in THF until the supernatant THF no longer produced a precipitate when dropped into water. The resulting gels were then dried in a vacuum oven at $100^{\circ} \mathrm{C}$.

Films of relative differing gel concentrations were formed by first allowing the gels to settle in graduated cylinders. Then for a film containing twice the normal gel concentration, the top half of the solution was drawn off and the bottom half was agitated to redisperse the gels. Films were then cast from these solutions. Differing relative concentrations of gels could also be obtained by drawing off more of the solution and redispersing the settled gels in the remaining solution.

Films were cast by adding a certain amount of a 2 percent solution cnto a specially made glass dish placed on a level surface. The film dish as well as a container of dessicant was covered with an enclosure that was nearly air tight so that the solvent would evaporate very slowly and in a dry environment. This method produced very good films, uniform in thickness and in clarity. The films produced were 1-3 mils in thickness. For plasticized films, the plasticizer was added before the separation of gels was accomplished.

\section{FILM APPEARANCE}

The most obvious consequence of the presence of the SPM gels in solution cast films was in surface topography and transparency. SPM-gel-free solutions produced perfectly clear, smooth, and transparent films for both the plasticized and unplasticized sample. Films cast from solutions containing the SPM gels became translucent due partly to the development of a definite, but very fine, surface texture that can be seen in Fig. 1. The texture was clearly apparent as well in plasticized films (Fig. 2). These photographs were taken with a Leitz MM5 Research Metallograph, which is a reflected light microscope.

The appearance of the film topography indicates that the SPM gels are associated or clumped to-

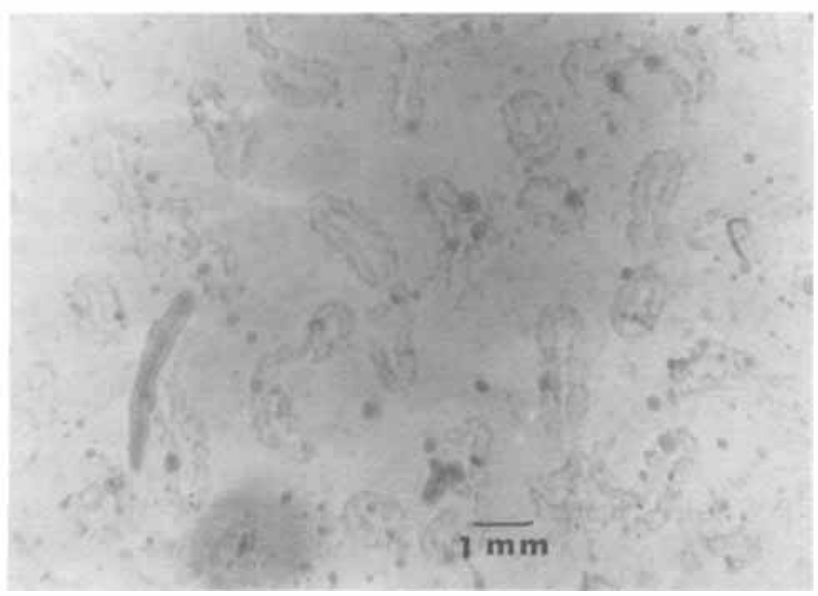

Fig. 1. Surface topography of unplasticized PVC solutioncast film containing approximately $\dot{0.4}$ percent unsoluble gels.

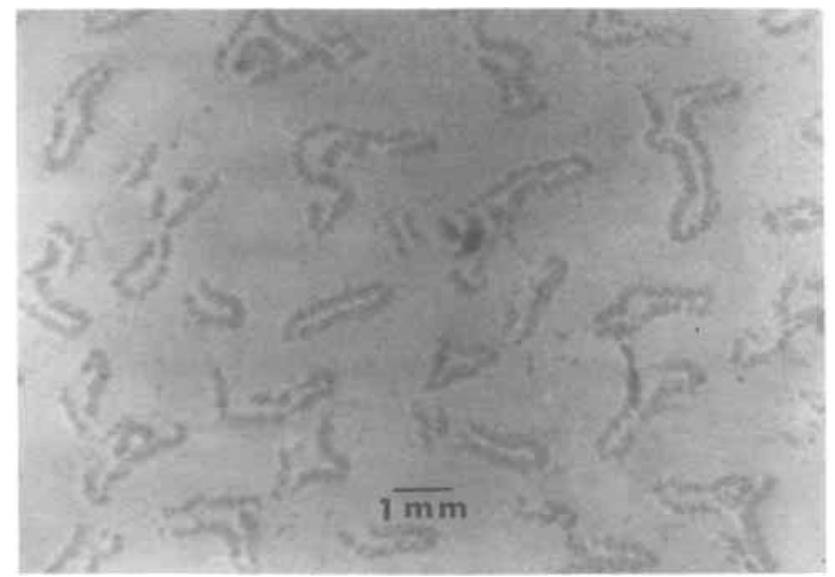

Fig. 2. Surface topography of PVC solution-cast fllm plasticized 20 percent with DOP containing 0.2 percent insoluble gels.

gether in the cast films. This is the dominant case, but for high concentrations of SPM gels this clumping did not always occur, as will be shown below.

The translucency of the films increases as the relative amount of SPM gels is increased, but never tó the extent that the films appear opaque. Films cast of concentrated SPM gel material were opaque but were impossible to handle since coherent films of this material could not be made. For subsequent IR measurements, the concentrated SPM gels had to be cast directly onto a $\mathrm{AgCl}$ crystal in order to obtain a spectrum.

One other situation was observed when the SPM gel concentration became relatively high, $\approx 1$ percent, which is shown in Fig. 3. Here a uniformly rough surface is observed. This may possibly be due to the observation that in solutions stored for relatively long times (six months), the suspension particles break down into the smaller particles that are described in the next section.

\section{INDIVIDUAL SPM PARTICLES}

When a purified suspension of SPM gels was dried on a glass microscope slide, irregular disc-like structures were observed of the order of $50 \mu \mathrm{m}$ in diam-

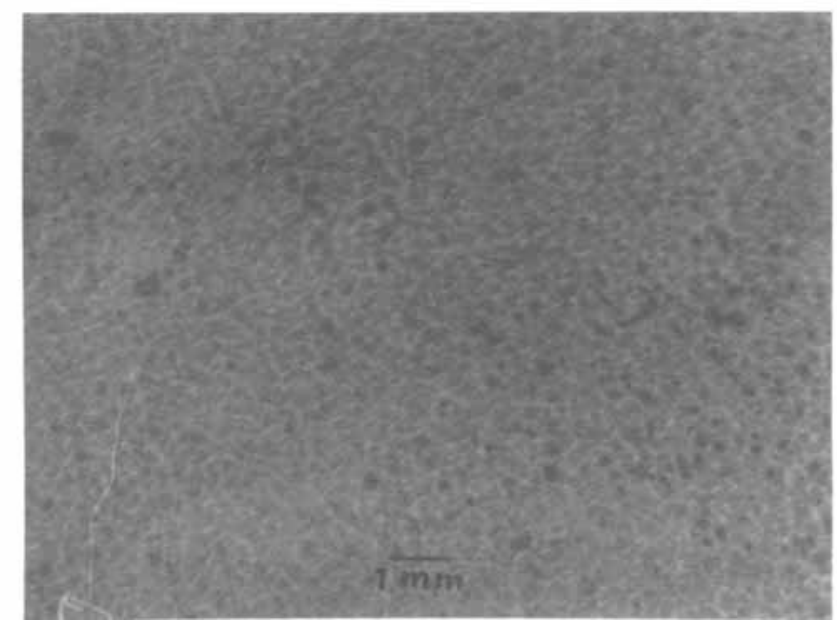

Fig. 3. Surface topography of unplasticized PVC film containing 1 percent insoluble gels. 


\section{F. E. Filisko}

eter (Fig. 4). The size of these particles is surprisingly uniform, irrespective of the source of the polymer from which the SPM particles were obtained. These particles could be ultrasonically dispersed or broken up into smaller particles which still remained insoluble. In SPM suspensions stored for long periods of time, however, it was observed that the SPM particles might spontaneously break down into small particles. Why this occurred is not known. The smaller particles still remained insoluble, though.

\section{HEATS OF SOLUTION}

Residual or structure-related heats obtained by subtracting the heat of mixing from the measured heats of solution (10) were obtained for both the bulk resins and resins with the gels removed. The results in Table 1 clearly show that the residual heats for samples with the gels removed are more negative or exothermic by $15-20$ percent. This indicates that the action of the solvent on the gels is such as to produce an endothermic reaction. An endothermic heat is evolved upon solution when the action of the solvent causes a destruction or break-up of ordered or crystalline regions. This indicates the possibility that upon separation of gels as described in the experimental section, not only the SPM gels were removed but also at least some of the crystalline aggregates; or that a significant amount of order/crystallinity exists in the SPM gels.

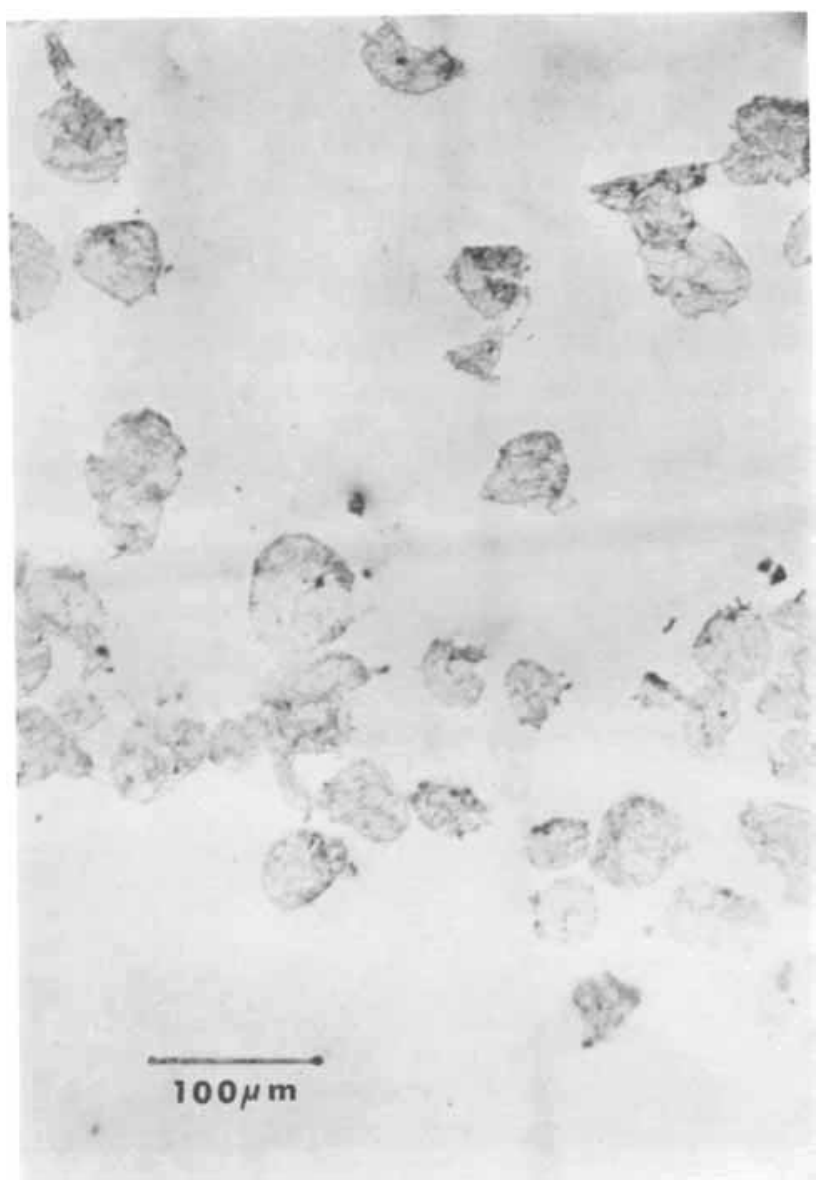

Fig. 4. Individual SPM gel particles.
Table 1. Residual Heats of PVC in THF and Cyclohexanone for the As-Received Resin and the Gel-Free PVC

\begin{tabular}{ccc}
\hline \multirow{2}{*}{$\begin{array}{c}\text { Molecular } \\
\text { weight }\end{array}$} & \begin{tabular}{c}
$\Delta \mathbf{H}_{\mathrm{r}}$, cal/gm \\
\cline { 2 - 3 } free
\end{tabular} & $\begin{array}{c}\text { As- } \\
\text { received }\end{array}$ \\
\hline 23,500 & -5.64 & -4.55 (in Cyclohexanone) \\
67,000 & -5.51 & -4.86 ((in Tetrahydrofuran) \\
138,000 & -6.57 & -5.45 \\
& -6.57 & -5.47 \\
& -6.68 & -6.08 \\
& -6.68 & -5.77 \\
\hline
\end{tabular}

Since it is known that the heats of solution of PVC in cyclohexanone and THF are exothermic (11) and the glassy heats are exothermic, then the difference is probably related to the destruction of order existing in the gels. This, however, is based on the assumption that all the gel material removed is completely PVC. Infrared measurements, however, indicate that the SPM gels consist only partly of $\mathrm{PVC}$ with the strong presence of both $\mathrm{OH}$ and $\mathrm{C}=\mathrm{O}$ groups present. If these groups are present in the form of polyvinyl alcohol and polyvinyl acetate, no strong thermal effects are expected, i.e., nowhere near the magnitude of the differences observed, since both these polymers are insoluble in both solvents used. However, the presence of these impurities in the gels removed still complicates matters since the interaction between these impurities and PVC is not known. In any case, it is interesting that such a small amount of material can make such a large difference in the measured heats of solution.

\section{INFRARED}

Infrared spectra were obtained on solution cast films of zero (Fig. 5A) and various gel concentrations, on concentrated gels (Fig. 5B), and on compression-molded films of the bulk PVC (Fig. 5C).

For concentrated gels (Fig. 5B) the spectra indicates a large and broad absorbance in the 3200$3500 \mathrm{~cm}^{-1}$ range, most likely due to $\mathrm{OH}$ stretching vibrations. Likewise, two large broad bands appear at about $1630 \mathrm{~cm}^{-1}$ and $1730 \mathrm{~cm}^{-1}$. These bands are probably due to $\mathrm{C}=\mathrm{O}$ and/or $\mathrm{C}=\mathrm{C}$ stretching vibrations (12).

In the bulk compression-molded samples of PVC the presence of these bands is clearly apparent. As the gel concentration in the films is increased, these bands also increase in intensity, implying that whatever mechanisms are responsible for the absorbances are specifically located in the gels.

The infrared spectra of pure gels (Fig. $5 B$ ) shows evidence of both PVC and PVOH with the exception that some bands characteristic of $\mathrm{PVOH}$ are decreased drastically in intensity. The 1630 and 1730 $\mathrm{cm}^{-1}$ absorbances do not appear in either the PVC or PVOH spectrum; however, they do appear in the polyvinyl acetate spectrum that may possibly be an impurity in the PVOH used as the suspending agent. Further, the gel spectra are not as sharp as pure $\mathrm{PVC}$ or $\mathrm{PVOH}$, indicating the presence of a struc- 
ture more complex than a simple mixture of PVC and PVOH. This is, however, not surprising considering the insolubility of the SPM gels.

Peaks in $600-700 \mathrm{~cm}^{-1}$ range indicative of syndiotacticity in PVC show no drastic differences between the bulk and concentrated gel material. It should, however, be mentioned that the spectra in this region are not very sharp, which is a consequence of the complex structure of these gels, thus possibly obscuring the presence of such bands. This
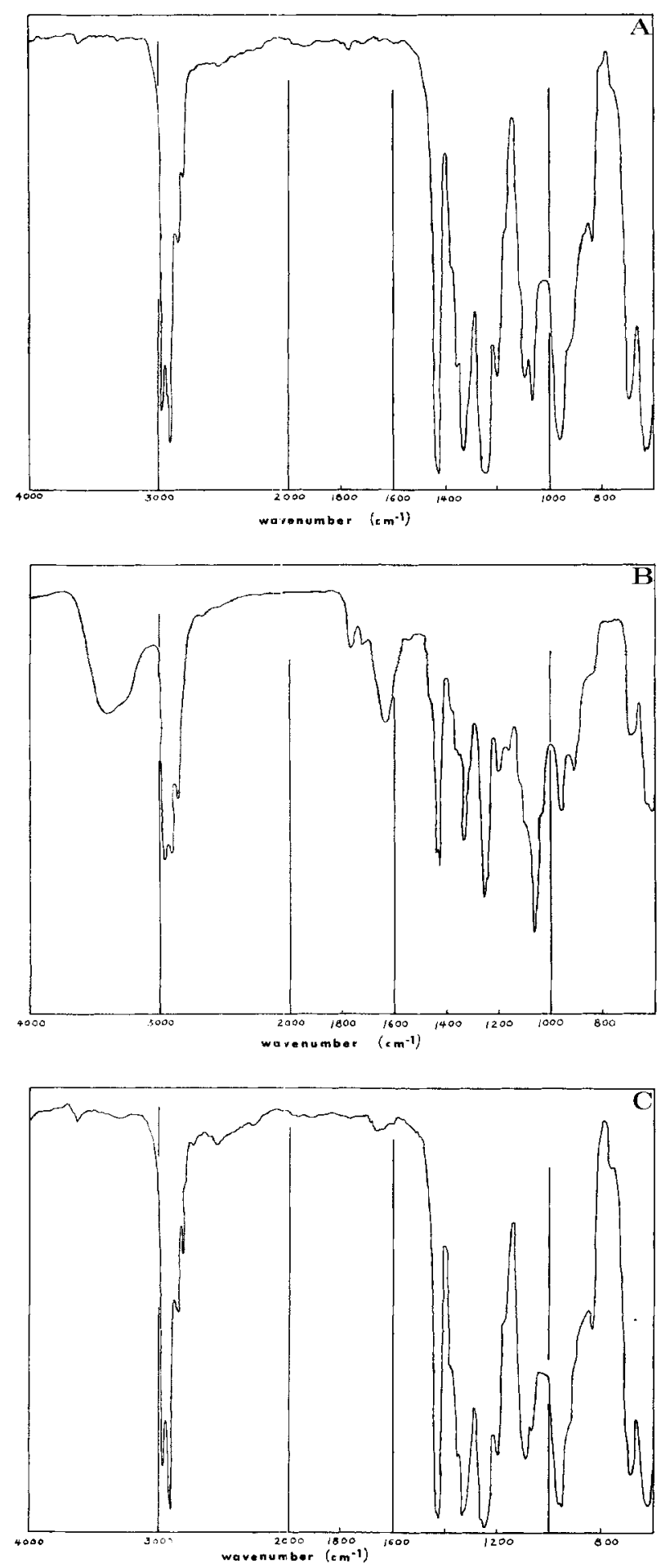

Fig. 5. Infrared spectrum of PVC solution cast films. A. all gels removed. $B$. concentrated gels. C. compression-molded bulk PVC. does not necessarily contradict the results of Vidotto and Bohdanecky (13) since their studies were performed on different types of samples and under different circumstances.

\section{ELECTRON MICROPROBE ANALYSIS}

A qualitative elemental analysis of the concentrated gels was performed in an attempt not only to obtain a rough idea of the amounts of $\mathrm{Cl}$ and $\mathrm{O}$ in the gels, but also to observe if either of these elements were concentrated solely on the SPM particles. The results, as shown in Table 2, clearly show the strong presence of chlorine both on the SPM and in the film residue material between these particles, approximately in the same relative concentration. More surprising was that oxygen appears not only on the SPM but also in the film residue between the SPM particles, and in nearly the same relative concentration. This indicates that the presence of oxygen either in the form of hydroxyl or carbonyl groups is found on the SPM as well as on other insoluble material or gels not of the SPE type. A large number of areas were observed with a $1 \mu \mathrm{m}$ diameter beam with the results being perfectly consistent.

\section{LOW-SPEED TENSILE MEASUREMENTS}

Low speed tensile measurements were performed with an Instron Universal Tester on four-inch long films of 0.214 inches width and thickness varying between 0.001 and 0.002 inches. Total elongation to break was not recorded due to difficulty in reproducing the fracture strains on these thin films. However, Young's Modulus, yield stress, and yield strain are shown in Table 3 for both the plasticized and unplasticized films.

Table 2. Electron Microprobe Analysis, Counts/10 sec

\begin{tabular}{lrc}
\hline & CI & D \\
\hline On SPM particles & $116 \pm 7$ & $64 \pm 10$ \\
Between particles & $67 \pm 8$ & $36 \pm 6$ \\
Substrate & $10 \pm 3$ & $16 \pm 3$ \\
\hline
\end{tabular}

Table 3. Modulus, Yield Stress, Yield Strain of Plasticized and Unplasticized Cast Films Versus Gel Concentration

\begin{tabular}{|c|c|c|c|c|c|c|c|}
\hline \multirow{2}{*}{$\begin{array}{c}\begin{array}{c}\text { Aggre- } \\
\text { gates, } \\
\text { percent }\end{array} \\
0 \\
0.2\end{array}$} & \multirow{2}{*}{$\begin{array}{c}\begin{array}{c}\text { DOP } \\
\text { content, } \\
\text { percent }\end{array} \\
0 \\
0\end{array}$} & \multicolumn{2}{|c|}{$Y \times \underset{p s i}{10-5}$} & \multicolumn{2}{|c|}{$\epsilon_{y}$, percent } & \multicolumn{2}{|c|}{$\sigma_{\mathrm{y}} \times \underset{p s i}{10-3}$} \\
\hline & & $\begin{array}{l}3.88 \\
4.24\end{array}$ & $\begin{array}{l}4.34 \\
4.08\end{array}$ & $\begin{array}{l}3.47 \\
3.31\end{array}$ & $\begin{array}{l}3.38 \\
3.18\end{array}$ & $\begin{array}{l}6.83 \\
7.31\end{array}$ & $\begin{array}{l}7.55 \\
7.25\end{array}$ \\
\hline $\begin{array}{l}0 \\
0.2\end{array}$ & $\begin{array}{l}4.0 \\
4.0\end{array}$ & $\begin{array}{l}4.76 \\
4.61\end{array}$ & $\begin{array}{l}4.60 \\
4.64\end{array}$ & $\begin{array}{l}3.27 \\
3.49\end{array}$ & $\begin{array}{l}3.31 \\
(2.21)\end{array}$ & $\begin{array}{l}8.55 \\
8.89\end{array}$ & $\begin{array}{l}8.75 \\
7.33\end{array}$ \\
\hline $\begin{array}{l}0 \\
0.2\end{array}$ & $\begin{array}{l}8.0 \\
8.0\end{array}$ & $\begin{array}{l}3.38 \\
3.17\end{array}$ & $\begin{array}{l}3.21 \\
2.79\end{array}$ & $\begin{array}{l}2.56 \\
2.62\end{array}$ & $\begin{array}{l}2.48 \\
2.27\end{array}$ & $\begin{array}{l}5.76 \\
5.09\end{array}$ & $\begin{array}{l}5.60 \\
4.65\end{array}$ \\
\hline $\begin{array}{l}0 \\
0.2\end{array}$ & $\begin{array}{l}12.0 \\
12.0\end{array}$ & $\begin{array}{l}2.86 \\
3.11\end{array}$ & - & $\begin{array}{l}2.72 \\
2.87\end{array}$ & $\overline{-}$ & $\begin{array}{l}4.55 \\
5.5\end{array}$ & - \\
\hline $\begin{array}{l}0 \\
0.4\end{array}$ & $\begin{array}{l}20.0 \\
20.0\end{array}$ & $\begin{array}{l}1.48 \\
1.23\end{array}$ & $\begin{array}{l}1.45 \\
1.39\end{array}$ & $\begin{array}{c}(3.70) \\
3.39\end{array}$ & $\begin{array}{l}3.75 \\
3.45\end{array}$ & $\begin{array}{l}2.86 \\
1.97\end{array}$ & $\begin{array}{l}2.64 \\
2.76\end{array}$ \\
\hline
\end{tabular}


Analysis of the data indicates that within experimental reproducibility no significant trends can be attributed to the presence of the SPM gels in the films, for both plasticized and unplasticized films. It must be remembered, however, that this data is obtained for solution-cast films which may be deceptive if attempting to extrapolate this data to molded or extruded samples.

What is not evident from the data is that the films always yielded by producing shear bands. In samples containing the SPM particles, it appeared that the bands could more easily form and the neck propagate further before rupture than for the SPMfree films. Thus the texture or roughness of the SPM containing films seems to facilitate the formation of shear bands.

High-speed tensile data (2500 percent/sec) was also obtained on PVC films, both plasticized and unplasticized, with various concentrations of the SPM particles. Again this data leads to no significant effect of the presence of these particles on the highspeed properties.

The above results appear puzzling since the incorporation of an inhomogeneity into a material would be expected to affect mechanical properties. However, no effect is observed in solution cast films. This behavior can be rationalized by noting that the SPM, from their nature would not be expected to function as an impact modifier. Further, as a result of the method of preparation, the SPM's cluster and do not form a continuous network structure throughout the film as might be expected in films prepared from virgin material by compression molding. Further studies are necessary to determine the role of the SPM and the effect of processing on the mechanical properties of PVC.

\section{SUMMARY AND CONCLUSIONS}

Insoluble components in solutions of suspensionpolymerized PVC consist of suspension particle membranes and crystalline aggregates. Although present in relatively small amounts $(\sim 0.1$ percent $)$ the membranes drastically affect the surface topography and clarity of solution-cast films. Heat of solution measurements indicate that the insoluble components removed by settling or centrifugation con- tain a significant amount of order or crystallinity. Infrared measurements indicate the presence of $a b$ sorption bands in the insoluble material is most likely due to the presence of hydroxyl and carbonyl groups that exist solely in the insoluble residue. These absorptions are probably due to the presence of poly(vinyl alcohol) used as the suspension agent and poly(vinyl acetate) impurities in the poly(vinyl alcohol). Elemental analysis of the residue indicates the presence of some oxygen and a large amount of chlorine on the suspension particle membranes as well as in the residue other than the membranes. Thus the residue seems to consist primarily of PVC with evidence of poly (vinyl alcohol) and poly (vinyl acetate) existing in a complex network structure. Finally the presence of insoluble components, primarily the suspension particle membranes, has no effect on the low-speed tensile and impact properties of the solution cast PVC films. The same results hold for films plasticized up to 20 percent with diethyl hexyl phthalate.

Acknowledgment is made to the donors of the Petroleum Research Fund of the American Chemical Society for support of this research.

\section{REFERENCES}

1. M. Bohdanecky, J. Polymer Sci., A2, 343 (1967).

2. M. Freeman and P. Manning, J. Polymer Sci., A2, 2017 (1964).

3. M. Kolinsky, J. Polymer Sci., C16, 485 (1967).

4. R. Tregan, A. Bonnemayre, and R. Tertian, Fifth International Congress For Electron Microscopy, 1, BB-10, Academic Press, New York (1962).

5. S. Maron and M. S. Lee, J. Macromol. Sci, B7, 29 (1973).

6. S. Maron and M. S. Lee, J. Macromol. Sci., B7, 47 (1973).

7. S. Maron and M. S. Lee, J. Macromol. Sci., B7, 61 (1973).

8. A. Berens and V. Folt, Trans. Soc. Rheol., 11, 195 (1967).

9. A. Berens and V. Folt, Polym. Eng. Sci., 9, 27 (1969).

10. S. Maron and F. Filisko, "Analytical Calorimetry," Porter and Johnson, editors, Plenum (1968).

11. S. Maron and F. Filisko, J. Macromol. Sci., B6, 413 (1972).

12. Personal communication, S. Krimm, University of Michigan, Ann Arbor.

13. G. Vidotto, Makromol. Chemie, 146, 159 (1971). 